\title{
GW23-e0560 A MODIFIED REGIMEN OF EXTRACORPOREAL CARDIAC SHOCK WAVE THERAPY (CSWT) FOR TREATMENT OF CORONARY ARTERY DISEASE
}

doi:10.1136/heartjnl-2012-302920j.48

Wang Yu, Cai Hongyan, Ma Teikun, Gao Tao. The First Hospital of Kunming Medical University

Objectives Our purpose was to evaluate the clinical outcomes of a new cardiac shock wave therapy (CSWT) treatment regimen with a shorter duration.

Methods A total of 55 patients with severe coronary artery disease $(\mathrm{CAD})$ were randomly divided into treatment group $\mathrm{A}(\mathrm{n}=20)$ and group $B(n=21)$ and control groups $(n=14)$. The control group received only medical therapy. In group A patients were treated for 1 week out of 4 , and CSWT was performed 3 times on days 1, 3, and 5 . The total duration of treatment was 3 months. In group $B$, patients underwent 3 CSWT sessions/week, and nine treatment sessions were completed within 1 month. Primary outcome measurement were 6-min walk test (6MWT). Other measurements like Canadian Cardiology Society (CCS) grading of angina, New York Heart Association (NYHA) functional classification, Seattle Angina Questionnaire (SAQ), nitroglycerin dosage (times/week), regional wall motion, peak systolic strain rate (PSSR), myocardial perfusion imaging (MRI) score were also evaluated.

Results After following for 12 months, the 6MWT, CCS grading of angina, dosage of nitroglycerin, NYHA classification, and SAO scores were all significantly improved in group $\mathrm{A}$ and $\mathrm{B}$ compared to control group. Group A and B also showed significant improvement over control group in peak systolic strain rate (PSSR) and myocardial perfusion imaging (MPI) under resting and load conditions. There was no significant difference in 6MWT between group $A$ and $B$ as well as other measurements.

Conclusions A CSWT protocol with 1 month treatment duration showed similar therapeutic efficacy compared to a protocol of 3 months duration. 